\title{
LA CALIDAD EN LA REHABILITACIÓN DE EDIFICIOS DESDE LA PERSPECTIVA DE LA CONSERVACIÓN DE LAS MADERAS ESTRUCTURALES Y DECORATIVAS
}

\author{
(RESTORATION QUALITY UNDER THE POINT OF VIEW OF THE STRUCTURAL \\ DECORATIVE WOOD ELEMENTS)
}

Angeles Navarrete de Varela*, Dr. Ing. de Montes

\section{RESUMEN}

La preocupación por la calidad de las rehabilitaciones de los edificios pertenencientes al Patrimonio Cültural Español, y las dificultades expuestas anteriormente para conseguirla, han llevado al Ministerio de Cultura a la elaboración del presente documento que sirviera de orientación a los arquitectos sobre el contenido que deben tener los informes previos a realizar por los expertos en maderas, en cuanto a las características mecánicas, estado de conservación, riesgos de degradación y tratatamientos de protección a aplicar a los distintos tipos y usos de maderas que se encuentran en un edificio.

\section{SUMMARY}

Preoccupancy for quality in rehabilitation of buildings wich belongs to the Spanish Cultural Patrimony and the difficulty to get it, is the reason to elaborate this document destinated to help architects and other experts about the content of technical informs to do.

Mechanics characteristics, state of conservation, degradation risks and protective treatemens are the main subjects os this work.

\section{INTRODUCCIÓN}

En los países desarrollados existe una preocupación, cada vez mayor, por la calidad de los productos que se producen. Esta preocupación por la calidad se ha hecho extensiva, en los últimos años, a la edificación, habiendo dado lugar a la elaboración y publicación de una "Directiva Comunitaria (89/106/CEE del Consejo del 21 de diciembre de 1988) sobre los productos de la construcción, entre ellos la madera y sus productos derivados y los productos que la protegen contra la acción de los agentes degradadores.
Como consecuencia de ella, los Comités Europeos de Normalización relacionados con los productos de construcción han recibido una orden formal de elaborar, con la mayor rapidez posible, todas las normas que permitan controlar la calidad de los materiales utilizados en la construcción.

La aplicación de estas normas, podrian, visto de una manera muy simplista, asegurar la calidad de cualquier obra arquitectónica nueva, pero no resolvería el problema de las obras de rehabilitación.

* Jefe de Grupo de Protección CIT-NIA Departamento de Industrias Forestales. Ministerio de Agricultura, Pesca y Alimentación. ESPAÑA 
En este caso no se pueden elegir los materiales de acuerdo con una norma de calidad, el arquitecto tiene que adaptarse a lo ya existente, cimentaciones, muros, estructuras, etc., y aunque se están llevando a cabo muchas investigaciones sobre nuevas técnicas para la realización de ensayos no destructivos (rayos $\mathrm{X}$, ultrasonido,...) todavía se está muy lejos de establecer ningún tipo de norma que permita determinar la calidad de la madera puesta en obra.

Por otra parte, si para obtener un producto arquitectónico de calidad se debe partir de un proyecto de calidad y según D. Mackay "un proyecto sólo puede ser de calidad si todas sus partes son completamente coherentes con la forma y el estilo de la arquitectura" el arquitecto, a la hora de realizar un proyecto de rehabilitación, tiene que conocer perfectamente el elemento del que parte y las características y el estado de los materiales de que está constituido.

En el caso que nos ocupa, centrándonos en el material "madera", tanto en funciones estructurales como decorativas, el único medio de que dispone el arquitecto para conocer bien sus características, estado de conservación, medidas de protección a tomar, etc., es la petición de unos estudios previos realizados por es. pecialistas.

La preocupación por la calidad de las rehabilitaciones de los edificios pertenecientes al Patrimonio Cultural Español y las dificultades expuestas anteriormente para conseguirla, han llevado al Ministerio de Cultura, a través del Instituto para la Conservación y Restauración de Bienes Culturales (I.C.R.C.B.) a pedir a la autora del presente trabajo, como especialista en Conservación y Protección de Maderas, su colaboración, dentro del Marco del Convenio existente entre el Ministerio de Cultura y el Instituto Nacional de Investigaciones Agrarias (INIA), para la elaboración de un documento que sirviera de orientación a los arquitectos sobre el contenido que deben tener los informes previos a rea-

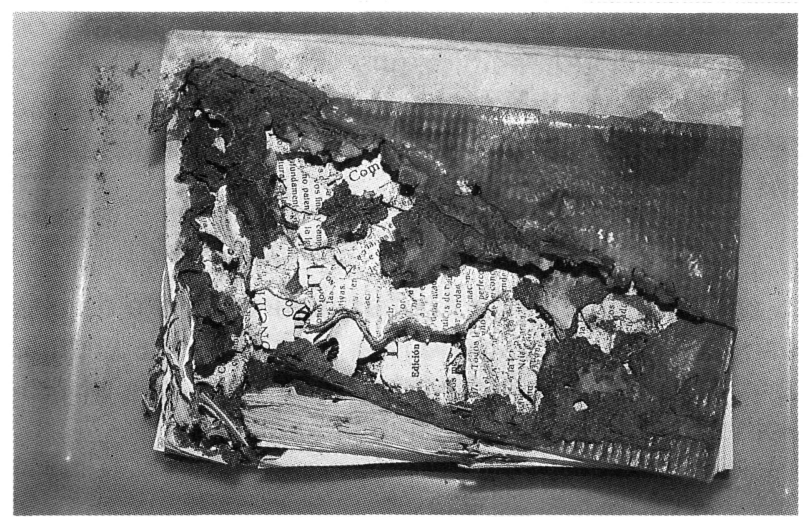

Libro procedente de la Iglesia de Castellar de la Frontera (Cádiz), in tensamente atacado por Reticulitermes lucifugus. lizar por los expertos en maderas, en cuanto a las características mecánicas, estado de conservación, riesgos de degradación y tratamientos de protección a aplicar a los distintos tipos y usos de maderas que se encuentran en un edificio.

La experiencia adquirida a través de mis años de profesión en los que he elaborado numerosos informes técnicos y las conversaciones y cambio de impresiones mantenidos con numerosos arquitectos, me han llevado a la conclusión de que para facilitar al arquitecto la redacción de la memoria del proyecto, estos estudios previos deben proporcionarle toda la información posible sobre las características mecánicas de las maderas, y grado de conservación, agentes destructores presentes en el edificio y condiciones que favorecen su desarrollo, medidas correctoras de protección y conservación a aplicar, es decir, deben seguir unas normas generales.

\section{NORMAS GENERALES PARA LA REDACCIÓN DE LOS ESTUDIOS DE PROTECCIÓN Y CONSERVACIÓN DE MADERAS EN EDIFICIOS A REHABILITAR}

Los Estudios previos deberán surninistrar al proyectista toda la información necesaria para poder:

- decidir, en función de las exigencias de uso futuro, ia sustitución o mantenimiento de las diferentes piezas de madera;

- planificar las medidas correctoras de tipo constructivo que ayuden a eliminar del edificio los organismos xilófagos que lo atacan y a rebajar la categoría de riesgo (Norma UNE 56.417) de las maderas puestas en servicio;

- elegir el tratamiento químico de protección que asegure la paralización de los ataques existentes y deje el edificio protegido contra posibles ataques futuros.

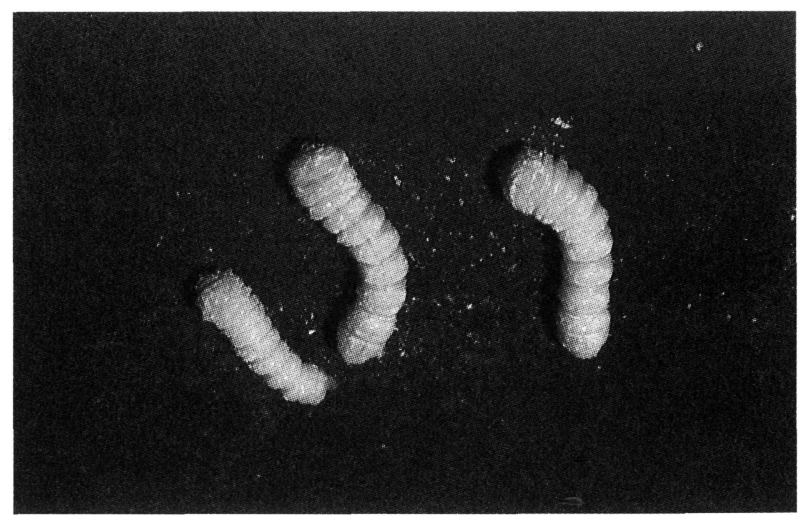

Larvas de Hylotrupes bajulus (Carcoma grande) en la última etapa de su desarrollo. 
Para ello, en estos estudios preliminares se consignarán: las especies de madera y agentes destructores presentes en el edificio, la localización y evaluación de los daños y, en función de ello y de las características propias del inmueble, se recomendará el tratamiento químico de protección que se considere más adecuado.

\section{1.- IDENTIFICACIÓN DE LAS MADERAS}

El conocimiento del género de madera nos permitirá determinar las características físico-mecánicas, imprescindibles para el cálculo de las tensiones admisibles, así como su susceptibilidad al ataque de los diferentes organismos xilófagos y su impregnabilidad con los productos protectores.

\section{2.- IDENTIFICACIÓN DE LOS AGENTES DESTRUCTORES}

Cada agente destructor requiere unas condiciones específicas para su desarrollo, lo que conlleva una estrategia de defensa propia para cada uno de ellos.

Los distintos agentes se van a detectar a través de los daños que han producido, diferenciándose entre ataques vivos o muertos, que indican que existen o han existido las condiciones adecuadas para el desarrollo de dicho agente, condiciones que, aunque en algunos casos hayan desaparecido, podrían repetirse, si no se actúa de forma adecuada, dando origen a nuevos ataques.

Los agentes destructores se dividirán en:

\section{1.- Agentes destructores bióticos}

2.1.1.- Hongos

2.1.1.1. - Mohos

2.1.1.2. - Hongos cromógenos

2.1.1.3. - Hongos de pudrición

2.1.2. - Insectos

2.1.2.1. - Insectos de ciclo larvario

2.1.2.2. - Insectos sociales

2.1.2.3.- Otros

\section{2.- Agentes destructores abióticos}

\section{3.- LOCALIZACIÓN Y EVALUACIÓN DE LOS DAÑOS}

La localización y evaluación de los daños, aportarán información sobre la resistencia mecánica de la estructura y permitirán establecer las medidas constructivas puntuales que modifiquen las condiciones de desarroIlo de los agentes presentes.

(c) Consejo Superior de Investigaciones Científicas Licencia Creative Commons 3.0 España (by-nc)
La evaluación de daños se realizará en las maderas de todo el edificio, poniendo especial atención en las zonas consideradas de alto riesgo, es decir, las próximas a fuentes de humedad.

Estas fuentes de humedad se pueden considerar divididas en:

- Pluviales: Goteras de cubierta, ventanas, limas, canalones y bajantes y humidificación de muros por azote de las lluvias o salpicaduras.

- Redes de distribución de agua: Conduciones a presión en zonas de servicios, red de conducciones de aguas residuales, circuito de calefacción por agua caliente.

- Humedades de capilaridad.

- Humedades de condensación.

La cuantificación de los daños se llevará a cabo a través de la determinación, por el método visual de las tensiones admisibles de clasificación, en función de los daños observados:

- Desviación de la fibra.

- Fendas y acebolladuras.

- Nudos.

- Gemas.

- Fibras rotas.

- Daños producidos por organismos xilófagos.

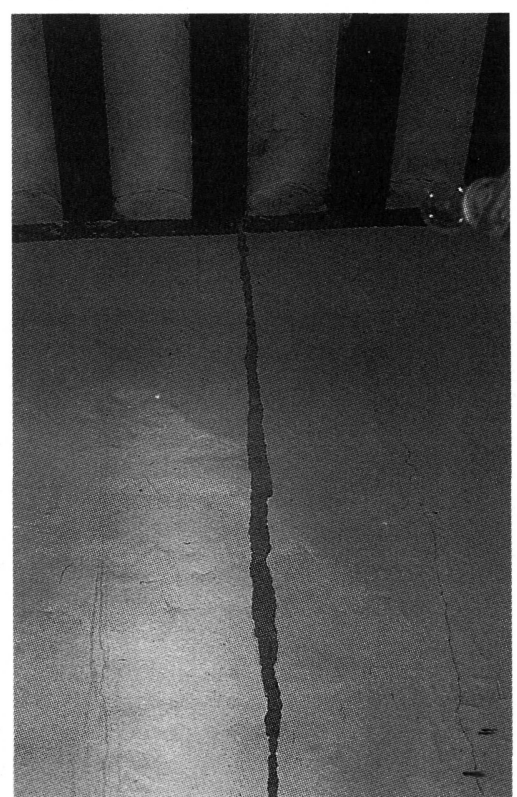

Construcción terrosa de termitas sobre una pared del Palacio del Nuevo Baztán (Madrid).

http://informesdelaconstruccion.revistas.csic.es 


\section{TRATAMIENTOS QUÍMICOS DE PROTECCIÓN Y CONSERVACIÓN}

Los tratamientos químicos de protección son específicos, en cuanto a las características y propiedades del producto a utilizar y en cuanto a su forma de aplicación, para cada agente destructor. Igualmente la estrategia a seguir es diferente para cada tipo de edificio y cada obra de rehabilitación.

Por todo ello habrá que estudiar el tratamiento contra cada uno de los agentes destructores presentes y la posible combinación entre todos ellos, en función de las características propias del edificio a rehabilitar.

Por consiguiente, en el estudio se deberá recoger cuál es el tratamiento o la combinación de tratamientos más adecuados, en función de los factores que intervienen en el edificio a rehabilitar.

Los tratamientos posibles serán los siguientes:

\subsection{Tratamientos contra agentes bióticos}

4.1.1.- Tratamientos contra hongos en maderas estructurales no decorativas.

4.1.2. Tratamientos contra Insectos:

4.1.2.1.- Tratamiento contra insectos de ciclo larvario en maderas estructurales no decorativas.

\subsubsection{2. - Tratamiento contra insectos sociales.}

Los tratamientos curativos contra las termitas subterráneas del género Reticulitermes, son particularmente delicados, como consecuencia de la forma de vida de estos insectos y del carácter extremadamente difuso de sus colonias. Normalmente, es imposible eliminar la totalidad de las colonias que atacan a un edificio y que están localizadas fuera de él.

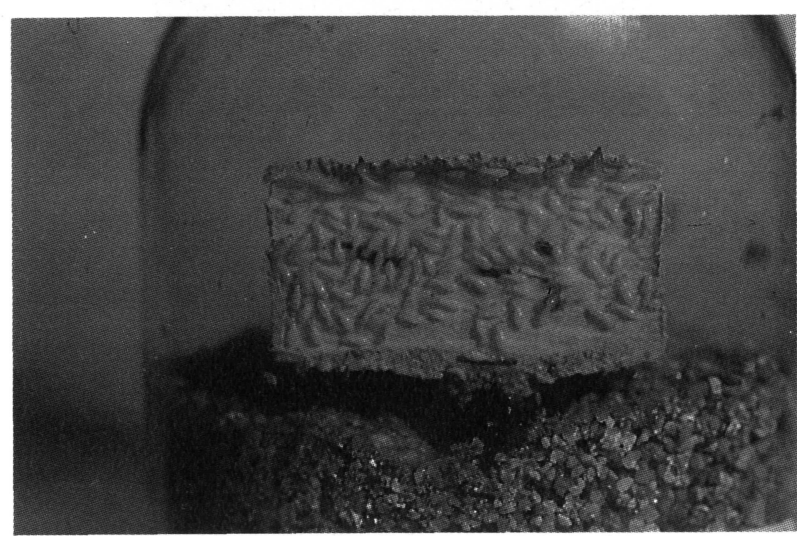

Termitas subterráneas de la especie Reticulitermes lucifugus.

(C) Consejo Superior de Investigaciones Científicas Licencia Creative Commons 3.0 España (by-nc)
Por consiguiente, la lucha contra las termitas se centra en impedir que continúe el ataque, aislando la construcción del medio exterior y destruyendo las colonias que se encuentran en el interior.

El tratamiento curativo de un edificio contra las termitas es más o menos difícil, dependiendo de las características propias del inmueble y de la intensidad y extensión del ataque.

Para obtener los mejores resultados, la lucha contra las termitas se realizará mediante la combinación de cuatro tratamientos diferentes que serán recomendados en el estudio previo, de acuerdo con los condicionantes expresados.

\section{Estos cuatro tratamientos son:}

A) Tratamiento de las funciones (Barrera química de protección).

B) Tratamiento de la base de los muros.

C) Tratamiento de otra parte de los muros.

D) Tratamiento de las maderas.

Si la complejidad del inmueble es grande, en el estudio se pueden proponer otros tratamientos complementarios.

4.1.3.- Tratamiento de las maderas decorativas o estructurales decoradas

4.1.4. Tratamiento de las maderas de reposición:

4.1.4.1.- Maderas estructurales.

4.1.4.2.- Carpintería.

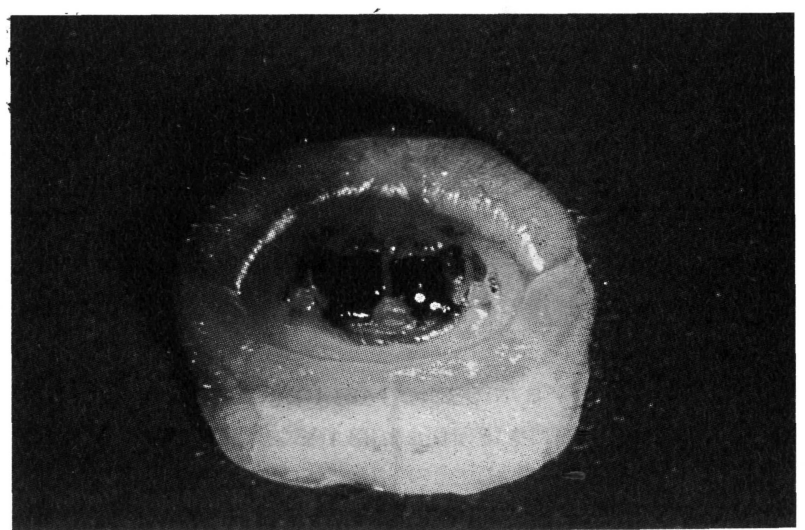

Potentes mandibulas de larva de carcoma grande.

http://informesdelaconstruccion.revistas.csic.es 


\subsection{Tratamiento contra agentes abióticos}

Dentro de los agentes abióticos se tendrán en cuenta exclusivamente los agentes meteorizantes (Iluvia, viento y sol).

No se incluirá el tratamiento contra el fuego, que deberá ser objeto de un estudio independiente.

Una vez que el arquitecto dispone de toda la información recogida en el estudio previo, está en condiciones de decidir la sustitución de las piezas de madera que lo requieran y solicitar la realización del tratamiento químico de protección más adecuado, sin correr el riesgo de aplicar el más costoso para cubrir cualquier tipo de riesgo y quedarse tranquilo, o por el contrario aplicar uno que no cubra todos los riesgos existentes y que se reproduzcan los ataques de xilófagos al cabo de pocos años de finalizada la obra, como ocurre con relativa frecuencia.

Este tratamiento químico de protección se debe realizar de acuerdo con un "Pliego de Condiciones Técnicas" basado en los datos aportados por el "Estudio previo", y debe definir: el tipo de protector a utilizar, el método de aplicación y la dosis de producto adecuado.

Ahora bien, como estas tres variables a fijar son diferentes para cada uno de los agentes destructores que pueden encontrarse en un edificio en rehabilitación, estableceremos unas condiciones diferentes para cada uno de dichos agentes destructores, así como para la combinación de los mismos, ya que rara vez actúan de forma aislada.

\section{PLIEGO DE CONDICIONES TÉCNICAS DEL TRATAMIENTO QUÍMICO DE PROTECCIÓN A EJECUTAR EN UN EDIFICIO EN REHABILITACIÓN}

\section{EDIFICIOS ATACADOS POR HONGOS}

\subsection{Tratamiento de maderas estructurales no deco- rativas.}

\subsubsection{Tipo de protector.}

Se utilizará un protector en disolvente orgánico de propiedades fungicidas y cuya eficacia haya sido contrastada por laboratorios oficiales (Normas UNE 56.415).

\subsubsection{Forma de aplicación.}

Una vez saneadas las zonas dañadas, se aplicará un tratamiento por inyección a presión a todas las piezas en que se hayan detectado pudriciones y tengan un espesor superior a $6 \mathrm{~cm}$, y a todas aquellas que, aunque no estén atacadas por hongos basidiomicetos, estén

(C) Consejo Superior de Investigaciones Científicas Licencia Creative Commons 3.0 España (by-nc) puestas en servicio en condiciones de riesgo 3 y 4 (maderas a la intemperie y en contacto permanente con una fuente de humedad. Norma UNE 56.417).

La distribución y diámetro de los taladros será tal que asegure la impregnación total de la pieza a tratar.

Las piezas de madera atacadas, de espesor menor de $6 \mathrm{~cm}$ y las sanas situadas en zonas de alto riesgo de humidificación (limas, zonas próximas a conducciones de agua, etc) recibirán un tratamiento superficial por pulverización o pincelado.

\subsubsection{Dosis del protector.}

La dosis a aplicar será la preconizada por el fabricante como eficaz para la forma de aplicación que vaya a practicarse (superficial o en profundidad), y la categoría de riesgo en que se encuentre, dentro del inmueble, la pieza a tratar.

\subsection{Tratamiento de maderas decorativas o estructu- rales decoradas.}

\subsubsection{Tipo de protector.}

Se utilizará un protector en disolvente orgánico de propiedades fungicidas y eficacia contrastada por laboratorios oficiales y que no afecte al aspecto estético de las maderas (Normas UNE 56.415).

\subsubsection{Forma de aplicación.}

El tratamiento se aplicará siempre que sea posible por la cara no decorada y únicamente a las piezas afectadas por pudrición.

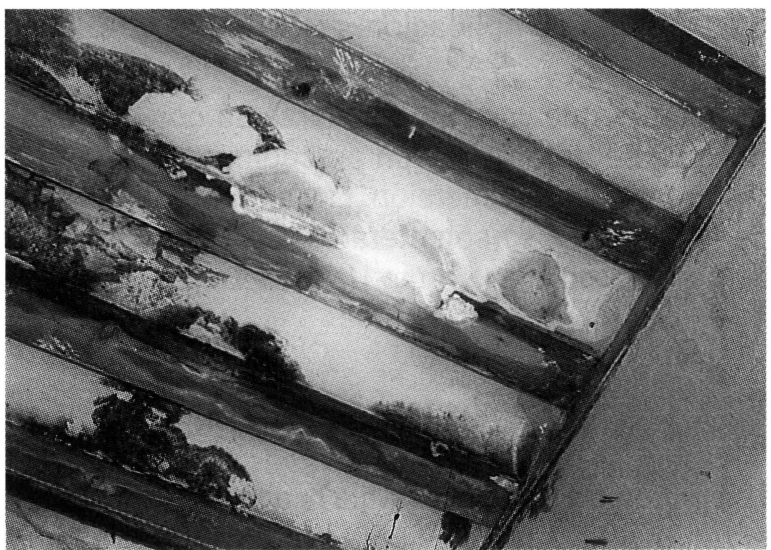

Cuerpos de fructificación de los hongos de pudrición que afectan a las viguetas de un forjado del Palacio del Nuevo Baztán (Madrid).

http://informesdelaconstruccion.revistas.csic.es 
Si las piezas de madera son de poco espesor, el protector se aplicará por pulverización o pincelado. Si el espesor de las piezas afectadas es superior a $6 \mathrm{~cm}$, el tratamiento se realizará por un procedimiento de impregnación profunda a baja presión y distribución del protector por capilaridad, a través de orificios de pequeño diámetro y en número y distribución tal, que aseguren la impregnación total de las piezas afectadas.

En el caso en que no sea accesible la cara no decorada, el tratamiento se realizará a través de la cara decorada, mediante el sistema de impregnación a baja presión descrito anteriormente.

\subsubsection{Dosis de protector.}

La dosis del protector será la preconizada por el fabricante como eficaz para la forma de aplicación que vaya a practicarse, superficial o en profundidad.

\subsection{Tratamiento de las maderas de reposición.}

\subsubsection{Tipo de protector.}

Para las maderas que vayan a estar situadas en zonas de riesgo 2 (maderas que accidentalmente alcanzan humedades por encima del $20 \%$ Norma UNE 56.417), se utilizará un protector en disolvente orgánico de propiedades fungicidas y eficacia preventiva contrastada por laboratorios oficiales (Norma UNE 56.415).

Para las maderas que vayan a estar situadas en condiciones de riesgo 3 ó 4 (madera a la intemperie o en contacto permanente con una fuente de humedad Norma UNE 56.417), se podrá utilizar un protector hidrosoluble no deslavable, de propiedades fungicidas contrastadas (Norma UNE 56.415).

\subsubsection{Forma de aplicación.}

Las piezas que vayan a colocarse en condiciones de riesgo 2 se tratarán por un procedimiento superficial, preferentemente inmersión. Las situadas en categoría de riesgo 3 y 4 se someterán a un tratamiento a presión en autoclave (Norma UNE 56.416).

\subsubsection{Dosis de protector.}

Los protectores se aplicarán a la dosis preconizada por el fabricante para el sistema de tratamiento elegido y la categoría de riesgo en que se vaya a encontrar la madera una vez puesta en servicio.

\section{EDIFICIOS ATACADOS POR CARCOMAS}

\subsection{Tratamiento de maderas estructurales no decorativas.}

\subsubsection{Tipo de producto.}

Para el tratamiento de la madera atacada se utilizará un producto en disolvente orgánico de propiedades insecticidas y cuya eficacia curativa haya sido contrastada por laboratorios oficiales (Norma UNE 56.415).

Para la madera de reposición se utilizará un protector en disolvente orgánico de propiedades fungicidas y eficacia preventiva (Norma UNE 56.415).

\subsubsection{Forma de aplicación.}

Para piezas de madera de espesor menor de $6 \mathrm{~cm}$ se aplicará por un procedimiento superficial (pulverización o pincelado) por una de sus caras.

Para piezas de madera de espesor entre 6 y $10 \mathrm{~cm}$, el protector se aplicará por un sistema superficial sobre sus cuatro caras, y si esto no fuera posible, por inyección profunda a través de una de sus caras, en taladros de diámetro, profundidad y distribución tales que aseguren la impregnación de toda la zona de albura de la pieza objeto del tratamiento.

Para piezas de madera de espesor superior a $10 \mathrm{~cm}$, el protector se aplicará por inyección a presión en taladros efectuados en la pieza de madera con una distribución, profundidad y diámetro que aseguren la impregnación de toda la parte de la pieza de madera susceptible de ser atacada.

La madera de reposición se tratará por un procedimiento superficial, preferentemente inmersión breve de 3 minutos.

\subsection{Tratamiento de maderas decorativas 0 estructurales decoradas.}

\subsubsection{Tipo de protector.}

Se utilizará un protector en disolvente orgánico de propiedades insecticidas y eficacia curativa contrastada por laboratorios oficiales (Norma UNE 56.415).

\subsubsection{Forma de aplicación.}

Se procederá en igual forma que en el apartado 1.2.2. 


\subsubsection{Dosis del protector.}

La dosis del protector será la preconizada por el fabricante como eficaz para la forma de aplicación que vaya a practicarse, superficial o en profundidad.

\subsection{Tratamiento de las maderas de reposición.}

\subsubsection{Tipo de protector.}

Se utilizará un protector en disolvente orgánico de propiedades insecticidas y eficacia preventiva contrastada por laboratorios oficiales (Norma UNE 56.415).

\subsubsection{Forma de aplicación.}

El protector se aplicará por un procedimiento superficial, preferentemente inmersión breve, y si no fuera po. sible, por pulverización o pincelado de todas sus caras.

\subsubsection{Dosis del protector}

La dosis del protector será la preconizada por el fabricante para la forma de aplicación que vaya a practicarse.

\section{EDIFICIOS ATACADOS POR TERMITAS SUBTERRANEAS}

\subsection{Tratamiento de las fundaciones (Barrera química de protección).}

\subsubsection{Tipo de protector.}

Se utilizará un producto de propiedades insecticidas, emulsionable en agua y no deslavable.

\subsubsection{Forma de aplicación.}

Se tratará el suelo, a una profundidad de 0,20 a $0,50 \mathrm{~m}$ de profundidad, alrededor de todo el edificio y lo más cerca posible de él, mediante la inyección del protector adecuado en taladros hechos cada 0,30-0,40 m según las características del terreno, o mediante la realización de una zanja de 0,20 a 0,50 $\mathrm{m}$ de profundidad y $0,50 \mathrm{~m}$ de ancho, regando con el protector el fondo y la tierra de relleno.

En el interior del edificio se inyectará el protector en el suelo, a una distancia de $0,40 \mathrm{~m}$ y a lo largo de todos los muros de carga, por el mismo procedimien. to que en la parte exterior.

\subsubsection{Dosis del protector.}

La dosis a utilizar será la preconizada por el fabricante como eficaz para la forma de aplicación seleccionada.

\subsection{Tratamiento de la base de los muros.}

3.2.1. Tipo de protector.

Se utilizará el protector descrito en el apartado 3.1.1.

\subsubsection{Forma de aplicación.}

Inyección del protector, a presión, en orificios taladrados sobre una línea horizontal situada a $0,40 \mathrm{~m}$ por encima del nivel del suelo, siempre por debajo del primer forjado, separados entre si 0,30-0,40 m, en función de las características del muro.

\subsubsection{Dosis del protector.}

La dosis a utilizar será la preconizada por el fabricante para este tipo de tratamiento.

\subsection{Tratamiento de otras partes de los muros.}

\subsubsection{Tipo de protector.}

Se utilizará un protector de las características descritas en el apartado 3.1.1.

\subsubsection{Forma de aplicación.}

Se realizará por inyección a presión del protector, justo por debajo de los forjados de madera y/o bajo la viga durmiente en que apoyan los pares de las cubiertas, si ésta es de madera, únicamente en las zonas de mayor riesgo, zonas en que la madera ha sido o puede ser atacada.

\subsubsection{Dosis del protector.}

La dosis a utilizar será la preconizada por el fabricante para este tipo de aplicación.

\subsection{Tratamiento de las maderas estructurales no decorativas.}

\subsubsection{Tipo de protector.}

Se utilizará un protector en disolvente orgánico de propiedades insecticidas y de eficacia probado contra las termitas (Norma UNE 56.415). 


\subsubsection{Forma de aplicación.}

Se impregnarán en profundidad, por inyección a presión, todas las vigas durmientes que estén en contacto con los muros y las cabezas de las viguetas de los forjados y pares de las cubiertas que apoyen sobre ellas, en las zonas en que se hayan detectado ataques o en las zonas de alto riesgo.

Igualmente se impregnarán en profundidad mediante inyección de insecticida los pies derechos que arranquen desde el suelo, hasta la altura del primer forjado.

En el caso de que el ataque de termitas haya avanzado por el interior de una viga, es necesario tratarla por inyección a presión en toda su longitud.

El diámetro, profundidad y distribución de los taladros será tal que asegure la impregnación total de la pieza a tratar, y variará en función de la especie de madera, de la escuadría de la pieza, proporción de albura y de duramen y número de caras descubiertas.

\subsubsection{Dosis del protector.}

La dosis a emplear será la preconizada por el fabricante para este tipo de tratamiento.

\subsection{Tratamiento de las maderas decorativas o estructurales decoradas.}

\subsubsection{Tipo de protector.}

Se utilizará un protector en disolvente orgánico de propiedades insecticidas, eficacia probada contra las termitas y que no dañe a las partes decoradas.

\subsubsection{Forma de aplicación.}

Siempre que sea posible, el tratamiento se aplicará por la parte no decorada en la forma descrita en el apartado 3.4.2. Si esto no es posible, los taladros que se practiquen en la zona decorada serán de pequeño diámetro.

Si las piezas a tratar son de espesores menores de 6 $\mathrm{cm}$, bastará un tratamiento superficial por pincelado o pulverización.

\subsubsection{Dosis del protector.}

Se utilizará la dosis preconizada por el fabricante como eficaz para la forma de aplicación elegida.

\subsection{Tratamiento de las tarimas.}

3.6.1. Tipo de protector. (Ver apartado 3.4.1.)

\subsubsection{Forma de aplicación.}

En el caso de que la tarima no se desmonte, tratar por pulverización la cara vista de las tablas.

Según sea la intensidad del ataque y el sistema constructivo, se completará el tratamiento, con una pulverización por debajo de la tarima, realizando algunas perforaciones a través de ella, en los espacios entre apoyos.

Si el ataque es muy intenso, o las viguetas de los forjados sobre las que se apoya la tarima no han podido tratarse desde el piso inferior, éstas se tratarán por inyección practicando taladros a través de la tarima, en la forma descrita en el apartado 3.4.2.

\subsubsection{Dosis del protector.}

La dosis de protector a aplicar, será la recomendada por el fabricante para este tipo de tratamiento.

\subsection{Tratamiento de las maderas de reposición.}

\subsubsection{Tipo de protector.}

Para las maderas estructurales, no decorativas, se utilizará un producto hidrosoluble de eficacia antitermitas y no deslavable.

Para las maderas decorativas se tendrá en cuenta el tipo de acabado que se les vaya a dar y la colocación que los protectores hidrosolubles comunican a la madera. Si estas dos cosas son compatibles, se utilizará el mismo producto descrito; si por el contrario no se puede variar el color de la madera se utilizará un protector en disolvente orgánico de propiedades antitermitas.

\subsubsection{Forma de aplicación.}

Para las maderas estructurales no decorativas y aquellas decorativas que admitan un cambio en su coloración, el tratamiento con el protector hidrosoluble se realizará a presión en autoclave.

Para las maderas decorativas que deban mantener su color, el protector orgánico se aplicará por inmersión (tiempo en función de las dimensiones de las piezas) o por doble vacío (Norma UNE 56.416).

\subsubsection{Dosis del protector.}

La dosis a aplicar será la preconizada por el fabricante para este tipo de tratamiento. 


\section{EDIFICIO ATACADO POR DOS O MÁS AGENTES DESTRUCTORES}

El tratamiento será la combinación de los tratamientos contra cada uno de los agentes destructores presentes, dando mayor importancia al destinado a combatir el agente destructor más dañino, que son las termitas subterráneas.

\subsection{Tipo de protector.}

Los protectores a utilizar deberán tener propiedades insecticidas y fungicidas y serán hidrosolubles o irán disueltos en un disolvente orgánico, según las piezas a tratar, y de acuerdo con lo expuesto en los tratamientos específicos descritos anteriormente.

\subsection{Forma de aplicación.}

La forma de aplicación será la descrita en el apartado correspondiente al agente destructor que afecta a cada pieza de madera, a sus características o al destino de la misma.

\subsection{Dosis de protector.}

La dosis de protector será la necesaria para combatir el agente destructor más dañino de los que se encuentren en el edificio.

\section{MADERAS ATACADAS POR AGENTES DESTRUCTORES ABIÓTICOS (METEORIZACIÓN)}

\subsection{Tipo de protector.}

Protector orgánico pigmentado de poro abierto y propiedades fungicidas e insecticidas. (Protector decorativo).

\subsection{Forma de aplicación.}

El protector se aplicará por un procedimiento superficial a las maderas decorativas (principalmente carpintería exterior) que estén sometidas a la acción de los agentes meteorizantes.

Este protector se aplicará a la madera en último lugar, es decir, después de que haya recibido los tratamientos curativos o preventivos que se hubiera previsto.

\subsection{Dosis del protector.}

La dosis será la recomendada por el fabricante y se aplicará como mínimo en dos manos.

\section{CONCLUSIÓN}

He tratado a lo largo de este trabajo de dar al arquitecto un punto de vista sobre el contenido que deben tener los "estudios previos"' sobre el estado y propiedades de las maderas, tanto estructurales, como decorativos, que a mi juicio proporciona toda la información que el arquitecto necesita para poder redactar eficazmente la memoria de un proyecto concreto de rehabilitación. Lo he completado con el pliego de condiciones técnicas del tratamiento químico de protección que debe ser específico para cada edificio, pues como ya hemos dicho varias veces, la problemática que entraña cada obra es diferente y por consiguiente hay que afrontarla de diferente manera.

Agradezco a la revista "Informes de la Construcción" la posibilidad de divulgar este trabajo y deseo que su lectura facilite a arquitectos y constructores la difícil labor de conseguir rehabilitaciones de calidad que contribuyan a conservar nuestro Patrimonio Cultural. 